\title{
Não linearidades, mudanças de regime e assimetrias na taxa de inflação brasileira: análise a partir de um modelo SETAR, 1944-2009 *
}

\author{
André M. Marques **
}

\begin{abstract}
Resumo
O objetivo principal deste estudo é investigar assimetrias e não linearidades na inflação brasileira, descrevendo-a como um processo autorregressivo sujeito a mudanças de regime. A metodologia empregada baseia-se na aplicação de testes para três tipos de não linearidade. Foi estimado um modelo SETAR com dois regimes capaz de descrever o comportamento não linear e assimétrico de um processo autorregressivo. Todos os testes indicaram a não linearidade da inflação brasileira. Os resultados das estimações sugerem a ocorrência de pelo menos dois regimes distintos para a inflação com diferentes processos geradores. O regime de baixa inflação é o menos volátil e o mais persistente. A despeito dos períodos de alta inflação e alta volatilidade, conclui-se que o regime de baixa inflação com baixa volatilidade é uma característica predominante na economia brasileira.
\end{abstract}

Palavras-chave: Inflação; SETAR; Não linearidade.

\section{Abstract \\ Applying SETAR model to detect some nonlinearities, change of regime and asymmetries in the brazilian inflation rate, 1944-2009}

The objective of this research is to investigate asymmetries, nonlinearities and regime changes in the Brazilian inflation rate in a long time span. The process is modeled employing a threshold time series model in order to take into account these parameters. The study was divided in two distinct stages. On the first stage, the Brazilian dataset was tested for several types of nonlinearities applying general and specific tests, e.g., quadratic and threshold behavior. On the second one, the Self-Exciting Threshold Autoregressive (SETAR) model was applied to indicate the threshold to which a signal of turning point could be provided in the states of the inflationary process. All the tests suggest that the Brazilian inflation rate is highly nonlinear and cannot be modeled as a linear and time reversible process. The results support the hypothesis that there are two distinct regimes with different generating processes and different volatilities and persistence. For instance, a lower inflation rate regime is more persistent with lower volatility.

Key words: Inflation; Threshold model; Brazil.

JEL C22, E31.

\section{Introdução}

As preocupações centrais de um policymaker em uma economia descentralizada podem ser crescimento, distribuição, equilíbrio externo,

\footnotetext{
* Trabalho recebido em 15 de fevereiro de 2010 e aprovado em 14 de junho de 2012

** Professor do Departamento de Economia da Universidade Federal da Paraíba, João Pessoa, PB, Brasil. E-mail: 00094751@ufrgs.br. Agradeço os valiosos comentários e sugestões de dois pareceristas anônimos. Erros e omissões que persistam são de inteira responsabilidade do autor.
} 
estabilidade de preços, entre outras. No entanto, em uma economia com alta inflação, essas diferentes metas dificilmente poderiam ser alcançadas pela política econômica. Por isso, a estabilidade de preços parece ser um problema chave para o formulador de política, de modo que a ocorrência de um regime de baixa inflação torna-se uma condição para se atingir os demais objetivos.

Na economia brasileira, desde a elaboração e implementação do Plano Real, em 1994, predomina uma baixa taxa de inflação anual. Apesar disso, há uma percepção corrente e bastante difundida de que a economia brasileira é predominantemente instável quando se tem em conta sua história pregressa. Nessa perspectiva, desde o período inicial da industrialização brasileira, mormente desde os anos 1950, uma alta taxa média de inflação parece predominar ao longo do tempo. Essa percepção emerge como senso comum na literatura.

Por exemplo, em um estudo abrangente sobre o assunto, em que vários casos de hiperinflação são examinados, incluindo a economia brasileira, Dornbusch et al. (1990, p. 2) observam que "While Cagan defined hyperinflation as an inflation rate of 50 percent per month (...) In particular, our threshold is a more modest (...), in Brazil, 15 percent per month". Para esses autores, a economia brasileira torna-se hiperinflacionária à medida que a taxa de inflação ultrapassa o limite de $15 \%$ ao mês.

Contudo, é necessário qualificar essa asserção teórica e examiná-la de modo mais sistemático. Será que uma taxa de inflação de $15 \%$ ao mês realmente separa a baixa inflação da hiperinflação no Brasil? Até o momento, apesar da disponibilidade de dados, nenhum estudo mais minucioso foi feito para analisar os elementos de verdade da afirmação feita pelos autores acima.

É verdade que vários autores têm estudado o comportamento da taxa de inflação brasileira para diferentes períodos históricos. No entanto, as análises realizadas até agora não apresentaram um teste explícito que levasse em conta possíveis não linearidades e mudanças de regime que a economia brasileira possa ter passado ao longo do tempo, desde 1944, quando o índice geral de preços mensal passou a ser calculado e disponibilizado pela primeira vez e o país experimentava a fase inicial de seu processo de industrialização.

Ademais, com base nos índices de preços da economia brasileira, há grande número de estudos que corroboram, em maior ou menor grau, a predominância da natureza inercial da inflação para vários períodos históricos ${ }^{1}$. Entre esses estudos, destacam-se Figueiredo e Marques (2009), Reisen et al. (2003), Cati et al. (1999), Campelo e Cribari-Neto (2003), Cribari-Neto e Cassiano (2005) e Maia e Cribari-Neto (2006). Laurini e Vieira (2005), por sua vez,

(1) Entre os principais trabalhos teóricos sobre a hipótese da inflação inercial, salientamos Lopes e Williamson (1980), Modiano (1983; 1985), Lopes (1985) e Resende (1985a, 1985b). 
enfatizam que a taxa de inflação no período pós Plano Real contém elementos de heterocedasticidade, especialmente em tempos de crises.

É importante ressaltar que os resultados apresentados por Laurini e Vieira (2005) e Figueiredo e Marques (2009) sugerem fortemente que a taxa de inflação brasileira é gerada por um processo não linear, por isso seria necessário um modelo adequado para se ter em conta essa característica de série. Até o momento, porém, não se tem notícia de um estudo que tenha realizado testes gerais e/ou específicos para a não linearidade da taxa de inflação no Brasil. Os autores acima apenas assumem essa característica em alguns de seus estudos e estimações. Antes de analisar em detalhe a série de inflação brasileira, algumas medidas descritivas são apresentadas, pois podem oferecer indícios de comportamento não linear, como a assimetria na distribuição dos dados.

O referencial teórico para interpretar a taxa de inflação como parte de um processo não linear tem sua base em publicações recentes que analisam a presença do caos e de vários tipos de não linearidade em séries econômicas (Pesaran; Potter, 1992). Nessa direção, Kiel e Elliott (2004, p. 5) observam que "Nonlinear systems may be characterized by periods of both linear and nonlinear interactions. During some time periods, behavior may reveal linear continuity. However during other time periods relationship between variables may change, resulting in dramatic structural or behavioral change".

Além disso, na literatura da inflação inercial, é bem conhecido o fato de que, quando a taxa de inflação se acelera, a demanda por indexação se eleva, realimentando o processo inflacionário (Lopes, 1985; Resende, 1985a, 1985b; Serrano, 2006). Assim, pode ser que haja um limiar (threshold) para a taxa de inflação que, uma vez ultrapassado, provoque uma intensificação do comportamento defensivo por parte dos agentes econômicos, resultando em um processo de hiperinflação dependente da trajetória. Exemplos de modelos teóricos formais, de caráter não linear, que descrevem um processo de hiperinflação separando regimes de baixa e alta inflação podem ser encontrados em Dornbusch et al. (1990) e Serrano (2006).

Mas, de uma forma geral, essa possibilidade teórica foi veiculada provavelmente pela primeira vez por Nicholas Kaldor quando observou que todos os casos conhecidos de hiperinflação (Alemanha, Hungria, Austrália e América Latina) têm a "(...) common characteristic that the rate of increase in prices reached the dimensions of a hyperinflation before it suddenly came to a halt." [But,] "Like the great plagues of the past they burnt themselves out". Segundo o autor, "(...) the explanation (...) is that the faster the inflation, the more prices and incomes become 'indexed' to inflation; and while the spread of indexing tends to accelerate inflation considerably, it also shortens the lag of adjustments of prices 
to costs, and of wages and salaries to prices; the smaller that lag, the weaker are the forces that keep the wage/price spiral going" (1982, p. 61). Essa asserção teórica foi formalizada por Lopes (1985) e fornece uma explicação para o fim de uma hiperinflação: à medida que o intervalo de reajustes de preços e salários tende para zero (de anual para semestral, trimestral para mensal, semanal para diário), o pico de renda real tende para o salário real médio e a inflação inercial deixa de existir.

Kaldor e Lopes notaram que esse mecanismo, em algum momento da hiperinflação, ocorre sempre que a moeda doméstica é substituída por outra unidade de medida sem inflação, como o dólar. Nesse momento, a nova unidade de medida usada pelos agentes está isenta da inflação que atinge apenas a velha moeda: a taxa de inflação é zero na nova moeda que circula na economia, enquanto a inflação corrente atinge somente a moeda anterior. Na nova moeda, a frequência de reajustes de preços e salários é zero. Esse processo, percebido por Pérsio Arida e André Lara Resende, levou à proposta do Plano Real, que, através da introdução de uma nova moeda indexada na economia, substituindo a moeda inflacionada (primeiro como unidade de medida, depois como meio de pagamento), gerou o fim do processo hiperinflacionário no Brasil. Essa é a interpretação do Plano Real segundo Delfim Netto (1997).

Por outro lado, não linearidade e teoria econômica são aspectos lógica e historicamente relacionados, e essa relação pode ser facilmente ilustrada pelo fato de que modelos não lineares (como o SETAR) podem conter e descrever dinâmicas endógenas, isto é, mesmo na ausência de choques exógenos sobre variáveis macroeconômicas, a série poderá flutuar de forma endógena, transitando entre um regime e outro. Isso está em forte contraste com o comportamento das séries temporais lineares, em que as flutuações são causadas inteiramente por choques exógenos e em que se supõe algumas propriedades estatísticas particulares sobre seu comportamento $\left(\varepsilon_{t}\right)$.

A implicação imediata para a política econômica a partir dessas observações é bastante clara. Uma vez que as flutuações da produção e/ou da inflação sejam endógenas, segue-se que políticas fiscais e monetárias ativas de administração da demanda agregada são uma necessidade lógica para se atingir e manter o pleno emprego da mão de obra e do estoque de capital com estabilidade de preços. Em contraste, na economia dos novos clássicos, em geral, supõe-se que a economia é assintoticamente estável, desde que não ocorram choques exógenos sobre as variáveis macroeconômicas. Se algumas variáveis macroeconômicas importantes apresentam dinâmica não linear, há boas razões para supor que o sistema econômico é inerentemente instável. Então, a mão visível do Estado será necessária para estabilizá-1o. Em estudo relacionado, Liu et al. (1992, S38) 
concluem que "Any economy is in theory essentially nonlinear in nature with complex interactions among many variables of economic significance".

Duas classes principais de modelos não lineares têm sido amplamente utilizadas. Os modelos de mudança de Markov (Markov-Switching), popularizados por Hamilton (1989), são vastamente empregados em análises do ciclo de negócios a fim de descrever as flutuações macroeconômicas. Franses e Djik (2003) oferecem estudo detalhado dessa abordagem. Nessa classe de modelos, supõe-se que o regime ou estado da natureza que vigora na economia não pode ser observado diretamente, porém é determinado por um processo estocástico subjacente não observável. Na segunda classe de modelos não lineares (SETAR e STAR), ao contrário, presume-se que os regimes ou estados da natureza vigentes na economia são determinados (caracterizados) por uma variável observada. Essa perspectiva será seguida neste trabalho ${ }^{2}$.

O modelo SETAR (Self-Exciting Threshold Autoregressive) introduzido por Tong e Lim (1980; Tong, 1983, 1990), pertence a esta última classe de modelos. Cryer e Chan (2008, cap. 15) analisam o caso típico desse modelo, e Franses e Dijk (2003, cap. 3) oferecem um estudo detalhado sobre a abordagem não linear de séries temporais, incluindo o SETAR e o modelo STAR (Smooth Transition Autoregressive).

Vários estudos recentes foram realizados utilizando os modelos SETAR e STAR. Potter (1995) estima um modelo SETAR para descrever o PIB dos EUA e apresenta evidências de efeitos assimétricos de choques ao longo do ciclo de negócios. Segundo o autor, o modelo estimado sugere que após 1945 a economia dos EUA é significativamente mais estável do que antes desse período. O mesmo modelo foi utilizado por Proietti (1998), assim como por Tiao e Tsay (1994), para descrever o PIB trimestral dos EUA. León-Ledesma (2006) estima um modelo SETAR para analisar o PIB dos EUA, da Alemanha e do Reino Unido.

Tendo como base esses estudos e aqueles relacionados com a taxa de inflação brasileira listados acima, a tarefa principal deste trabalho, seguindo Keenan (1985), Tsay (1986), Tong (1990) e Cryer e Chan (2008), é testar a série de inflação brasileira para vários tipos de não linearidades. Foram realizados testes gerais, sem alternativa específica e também com alternativa específica (do tipo threshold). Em seguida, foi empregada a metodologia mais recente do modelo SETAR para detectar o limiar para a taxa de inflação brasileira que determina a mudança entre os regimes ou estados do processo inflacionário. O modelo estimado é capaz de descrever não linearidades, assimetrias e mudanças de regime

(2) Essa seria a alternativa preferível em conformidade com a escola de Cambridge, em que os fatores observáveis e persistentes devem ser predominantes na análise econômica. Ver Eatwell e Milgate (1983) e Kaldor (1982) 
da taxa de inflação brasileira por um longo período de tempo, tendo em conta a heterocedasticidade dos dados.

Todos os testes realizados, em geral, sugerem que a taxa de inflação brasileira é altamente não linear e que não pode ser modelada como se fosse um processo linear e reversível no tempo. Os resultados indicam a ocorrência de dois regimes distintos com diferentes processos geradores, volatilidades e graus de persistência. O regime de baixa inflação é mais persistente e está associado à menor volatilidade.

O trabalho está organizado do seguinte modo. Após a Introdução, na primeira seção, são apresentados a base de dados, algumas medidas descritivas e gráficos que auxiliam a caracterizar seu comportamento. A segunda seção destaca a metodologia dos testes gerais e específicos para a não linearidade. A terceira seção descreve o modelo SETAR. A seção quatro avalia e discute os resultados alcançados. Na última seção são feitos os comentários finais.

\section{Descrevendo a taxa de inflação brasileira}

Algumas das principais características de uma série temporal não linear são as assimetrias das realizações, a presença de regimes diferentes (em que a média, a variância e a autocorrelação dependem do regime vigente) e a volatilidade em clusters (Franses; Djik, 2003, cap. 1). Assimetrias manifestam-se em seu comportamento cíclico, em que a série tende para valores negativos (ou próximos de zero) rapidamente, porém, para valores positivos (crescentes), de forma relativamente lenta. Essa característica está presente na taxa de inflação brasileira (ver figura 1, abaixo). Isso significa que a estrutura probabilística que caracteriza o processo gerador dos dados será diferente, uma vez que se inverta o sentido temporal da série ${ }^{3}$.

Além disso, a heterocedasticidade dos dados aparece em clusters, concentrada em diferentes períodos de tempo, refletindo a ocorrência de regimes distintos no longo prazo. Infelizmente, esta última característica não pode ser visualizada na Figura 1, abaixo, mas poderá ser estimada mais tarde. Essas propriedades foram estudadas em profundidade por Cryer e Chan (2008, cap. 15) e Franses e Dijk (2003).

Observando-se algumas medidas descritivas da série, pode-se ter uma percepção geral de sua distribuição. A Tabela 1, abaixo, apresenta um resumo das medidas descritivas da taxa de inflação brasileira de fevereiro de 1944 a novembro de 2009. Para expressar a taxa de inflação brasileira, como em Figueiredo e Marques (2009) e Reisen et al. (2003), foi utilizada a variação percentual mensal

(3) Séries temporais lineares são reversíveis no tempo. Ver Tong (1990). 
do IGP-DI, que é uma síntese de outros índices de preços. Essa é uma medida generalizada do processo inflacionário na economia brasileira.

Tabela 1

Medidas descritivas para a taxa de inflação - Brasil - IGP-DI- (\%) - 1944-2009

\begin{tabular}{c|c}
\hline Variável & IGP-DI \\
\hline $\mathrm{N}$ & 790 \\
\hline Mínimo & $-2,59$ \\
\hline Máximo & 81,32 \\
\hline Amplitude & 83,91 \\
\hline Média & 5,00 \\
\hline Mediana & 1,81 \\
\hline Desvio-padrão & 9,07 \\
\hline Assimetria & 3,7 \\
\hline Curtose & 17,83
\end{tabular}

Fonte: Elaboração própria.

A principal conclusão, a partir das informações acima, é de que a taxa de inflação não segue a distribuição normal, e que há grande assimetria nas observações. Uma vez que a mediana situa-se sensivelmente abaixo da média, é difícil atribuir distribuição normal para essa série. No que se refere à característica de assimetria, no caso normal, ela seria zero. Há evidências de que a maior parte das realizações efetivamente ocorrem na cauda esquerda da distribuição.

Quando se analisa a medida de curtose, constata-se que ela é muitas vezes maior que a do caso normal, cuja medida é 3 . Isso reflete o fato de que as caudas da distribuição dessa variável são mais pesadas que as caudas da distribuição normal. Em outras palavras, as grandes realizações da série ocorrem com mais frequência do que se poderia esperar em uma distribuição normal.

Com o auxílio de um gráfico específico, com a série temporal invertida, sugerido por Tong (1990), pode-se averiguar a existência de irreversibilidade no tempo do conjunto de dados e, assim, compreender melhor a estrutura probabilística de uma série temporal. Os dados são dispostos de modo inverso no tempo, em que o eixo das abscissas apresenta a sequência temporal invertida. A inspeção visual é feita comparando-se a trajetória normal da taxa de inflação brasileira na parte superior da figura $1 \mathrm{com}$ a sua trajetória na sequência temporal invertida (parte inferior).

Pode-se detectar imediatamente a irreversibilidade no tempo dos dados, uma vez que a trajetória da taxa de inflação não seria a mesma com o tempo invertido. $\mathrm{Na}$ trajetória normal, a taxa de inflação sobe lentamente e a cai abruptamente. Porém, com o tempo invertido, a inflação cresce rapidamente e 
decai lentamente. Esse fenômeno é conhecido, na literatura, como irreversibilidade do tempo ${ }^{4}$.

\section{Figura 1}

Trajetória normal da taxa de inflação (superior) e sequência temporal invertida (abaixo)

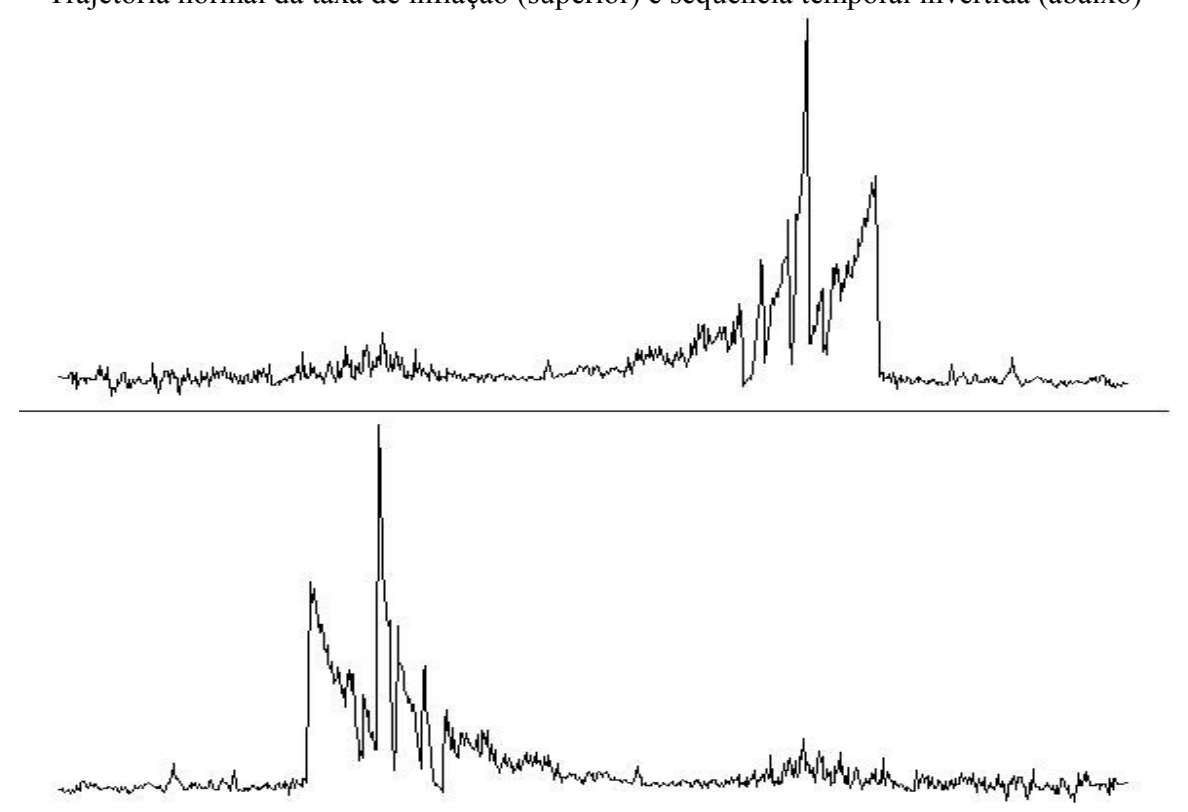

Fonte: Elaboração própria.

Ao observar a parte superior da Figura 1, constata-se que a taxa de inflação no Brasil tende a crescer mais lentamente do que quando está em queda. A fase crescente é mais lenta que a fase de queda, característica típica da trajetória não linear. Além disso, a figura sugere que, em alguns períodos específicos, a volatilidade da inflação aumentou em clusters, como na fase inicial de industrialização do país, em 1960 e 1990 e na segunda "década perdida" da economia brasileira.

As medidas descritivas fornecem indícios de comportamento assimétrico e de uma distribuição de caudas pesadas associada à inflação. Alguns testes de hipóteses para a não linearidade são necessários para verificar a necessidade de uma abordagem não linear para a série. Vários testes têm sido propostos para averiguar a não linearidade de uma série temporal. Neste trabalho, no entanto, segue-se a metodologia apresentada em Cryer e Chan (2008) e Franses e Dijk (2003). Três testes principais são discutidos em detalhe por esses autores.

(4) Para uma definição formal de irreversibilidade no tempo, ver Tong (1990). 


\section{Testes para não linearidade}

Keenan (1985) derivou um teste para não linearidade com uma alternativa específica. Para o autor, uma série de tempo não linear estacionária pode ser aproximada por uma expansão de Volterra de segunda ordem:

$$
\text { (1) } Y_{t}=\mu+\sum_{\mu=-\infty}^{\infty} \theta_{\mu} \varepsilon_{t-\mu}+\sum_{v=-\infty}^{\infty} \sum_{\mu=-\infty}^{\infty} \theta_{\mu \nu} \varepsilon_{t-\mu} \varepsilon_{t-v}
$$

onde $\left\{\varepsilon_{t},-\infty<t<\infty\right\}$ são variáveis i.i.d. com média zero. O processo $\left\{Y_{t}\right\}$ será linear se o duplo somatório do lado direito da equação (1) resultar em zero. A estatística de teste de Keenan será denotada por $\hat{F}_{K}$. Sob a hipótese nula de linearidade, a estatística $\hat{F}_{K}$ segue aproximadamente uma distribuição $F$ com 1 e $n-2 m-2$ graus de liberdade, onde $n$ é o tamanho da amostra e $m$ é a ordem de defasagem do modelo. Cryer e Chan (2008, p. 390-391) mostram que o teste de Kenan é equivalente à hipótese de $\eta=0$ no seguinte modelo de regressão:

$$
\text { (2) } Y_{t}=\theta_{0}+1+\phi_{1} Y_{t-1}+\ldots+\phi_{m} Y_{t-m}+\eta\left(\sum_{j=1}^{m} \phi_{j} Y_{t-j}\right)^{2}+\varepsilon_{t} \text {. }
$$

Contudo, Tsay (1986) expandiu o teste de Keenan para alternativas não lineares mais gerais e mostrou que é equivalente a considerar o seguinte modelo de regressão quadrática:

$$
\begin{array}{ll} 
& Y_{t}=\theta_{0}+\phi_{1} Y_{t-1}+\ldots+\phi_{m} Y_{t-m} \\
& +\delta_{1,1} Y_{t-1}^{2}+\delta_{1,2} Y_{t-1} Y_{t-2}+\ldots+\delta_{1, m} Y_{t-1} Y_{t-m} \\
& +\delta_{2,2} Y_{t-2}^{2}+\delta_{2,3} Y_{t-2} Y_{t-3}+\ldots+\delta_{2, m} Y_{t-2} Y_{t-m}+\ldots \\
& +\delta_{m-1, m-1} Y_{t-m}^{2}+\delta_{m-1, m} Y_{t-m+1} Y_{t-m}+\ldots+\delta_{m, m} Y_{t-m}^{2}+\varepsilon_{t}
\end{array}
$$

e testar se todos os $m(m+1) / 2$ coeficientes $\delta_{i, j}$ são zero. Novamente, isso pode ser feito pelo mesmo teste $F$ anterior. Para especificar o valor da defasagem $m$, na prática, o critério de informação AIC é utilizado. Outros testes podem ser aplicados para detectar não linearidades em séries temporais, como o teste BDS, baseado em conceitos da teoria do caos (Liu et al., 1992).

Se por um lado, os testes de Keenan e Tsay podem detectar não linearidades quadráticas, a não linearidade com alternativa específica do tipo threshold não pode, por outro, ser capturada pelos testes acima. Chan (1990) desenvolveu um teste de hipóteses com essa alternativa específica (Cryer; Chan, 2008). A hipótese nula do teste é de que a série é descrita por um processo $A R(p)$, 
e que a alternativa é um SETAR estacionário de ordem $p$ com resíduos normalmente distribuídos. $\mathrm{O}$ modelo geral para a hipótese alternativa pode ser escrito como

$$
\begin{aligned}
& Y_{t}=\phi_{1,0}+\phi_{1,1} Y_{t-1}+\ldots+\phi_{1, p} Y_{t-p} \\
& +\left\{\phi_{2,0}+\phi_{2,1} Y_{t-1}+\ldots+\phi_{2, p} Y_{t-p}\right\} I\left(Y_{t-d}>r\right)+\sigma e_{t}
\end{aligned}
$$

onde $I(\cdot)$ é uma variável indicadora equivalente à unidade se a expressão entre parênteses for verdadeira, e zero, caso contrário. O teste é realizado para valores fixos de $p$ e $d$ (parâmetro de defasagem) e mostra que é equivalente a obter

$$
\text { (5) } T_{n}=(n-p) \log \left\{\frac{\hat{\sigma}^{2}\left(H_{0}\right)}{\hat{\sigma}^{2}\left(H_{1}\right)}\right\} \text {, }
$$

onde $n-p$ é o tamanho efetivo da amostra, $\hat{\sigma}^{2}\left(H_{0}\right)$ é o estimador de máxima verossimilhança da variância dos resíduos do modelo linear $A R(p)$ estimado e $\hat{\sigma}^{2}\left(H_{1}\right)$ é proveniente do SETAR estimado com a threshold buscada dentro de um intervalo finito. O teste da razão máxima de verossimilhança descrito pela expressão (5) não segue uma distribuição padrão. Chan (1991) derivou um método de aproximação computacional para calcular as probabilidades exatas do teste de forma bastante precisa. O teste depende do intervalo fixado para que o parâmetro de threshold seja buscado. Seguindo Cryer e Chan (2008), neste estudo, foram adotados o $10^{\circ}$ e o $90^{\circ}$ percentil da amostra como limites.

Os resultados dos três testes descritos acima são apresentados a seguir. Em geral, pode-se inferir que a taxa de inflação brasileira não tem as propriedades de uma série de tempo linear e que um modelo $A R(p)$ não seria adequado para descrever seu comportamento.

Tabela 2

Resultados para não linearidades quadráticas

\begin{tabular}{c|ccc}
\hline \multirow{2}{*}{$\begin{array}{c}\text { Teste de } \\
\text { Keenan }\end{array}$} & $\hat{F}_{K}$ & $p$-valor & ordem de defasagem* \\
\cline { 2 - 4 } & 27,14 & 0,000 & 16 \\
\hline \multirow{2}{*}{ Teste de Tsay } & $\hat{F}_{T}$ & $p$-valor & ordem de defasagem* \\
\cline { 2 - 4 } & 20,94 & 0,000 & 16 \\
\hline
\end{tabular}

Nota: * selecionada a partir do critério do mínimo AIC.

Fonte: Elaboração própria. 
Tabela 3

Resultados para não linearidade do tipo threshold

\begin{tabular}{c|cccccc}
\hline$\hat{d}$ & 1 & 2 & 3 & 4 & 5 & 6 \\
\hline$\hat{T}_{n}$ & 112,302 & 125,011 & 69,011 & 37,625 & 50,889 & 62,275 \\
\hline$p$-valor & 0,000 & 0,000 & 0,000 & 0,000 & 0,000 & 0,000 \\
\hline
\end{tabular}

Fonte: Elaboração própria.

Todos os resultados apresentados acima sugerem que o processo gerador da inflação brasileira é altamente não linear. Nota-se que a maior estatística de testes para a não linearidade do tipo threshold é obtida quando $d=2$, mas $d=1$ é também competitiva ${ }^{5}$.

\section{Descrição do modelo que captura a mudança de regime}

A estimação do modelo SETAR é condicionada aos valores inciais $\max (p ; d)$, onde $p$ é a ordem do processo e $d$ é o parâmetro de defasagem. Se o parâmetro de threshold $r$ e o parâmetro de defasagem $d$ forem conhecidos, a base de dados pode ser dividida em duas partes (dois regimes) de acordo com a condição $Y_{t-d} \leq r$ ou $Y_{t-d}>r$. Para estimar o SETAR, foi utilizado o critério do mínimo AIC por meio do qual os parâmetros são estimados por mínimos quadrados ordinários em cada submodelo, seguindo a abordagem de Cryer e Chan (2008, p. 402-405). Primeiro, entretanto, busca-se obter a ordem mais adequada de degasagem de cada submodelo, fixando-se os valores $\max (p=5 ; d=5)$.

A bem da simplicidade, é descrito, abaixo, o modelo de apenas dois regimes. Contudo, o caso de $m$ regimes pode ser imediatamente obtido. Para isso, pode-se consultar Cryer e Chan (2008, cap. 15) e Franses e Dijk (2003, cap. 3). O processo estacionário $\left\{Y_{t}\right\}$ segue um SETAR com dois regimes denotado por $\operatorname{SETAR}\left(2, p_{1}, p_{2}\right)$ com parâmetro de defasagem $d$ se for descrito pela seguinte equação:

$$
\text { (6) } Y_{t}=\left\{\begin{array}{lll}
\varphi_{1,0}+\varphi_{1,1} Y_{t-1}+\ldots+\varphi_{1, p_{1}} Y_{t-p_{1}}+\sigma_{1} e_{t} & \text { se } & Y_{t-d} \leq r \\
\varphi_{2,0}+\varphi_{2,1} Y_{t-1}+\ldots+\varphi_{2, p_{2}} Y_{t-p_{2}}+\sigma_{2} e_{t} & \text { se } & Y_{t-d}>r
\end{array}\right. \text {, }
$$

onde $r$ é o parâmetro de threshold, $d>0$ é o parâmetro de defasagem, $e_{t}$ é um ruído branco. $I(\cdot)$ é uma função indicadora de que $I\left(Y_{t-d}>r\right)=1$ se $Y_{t-d}>r$ e zero, caso contrário. Para uma dada threshold $r$ e para uma conhecida posição da

(5) A estimação do modelo SETAR com $d=2$ foi realizada com AIC calculado em 3197, sensivelmente maior quando comparado à estimação do modelo com $d=1$, cujo AIC é 3128. 
variável aleatória $Y_{t-d}$ com relação à threshold $r$, o processo autorregressivo $\left\{Y_{t}\right\}$ segue um submodelo particular $A R(p)$. As ordens de defasagem $p_{1}$ e $p_{2}$ não serão necessariamente idênticas porque o critério de mínimo AIC determina a ordem de cada submodelo de acordo com a threshold e o parâmetro de defasagem d. Na prática, o algoritmo de estimação avalia um grande número de combinações de $r$ e $d$ (ver abaixo). Observe-se que todos os parâmetros são estimados simultaneamente, buscando-se o melhor SETAR, e o parâmetro $d$ poderá ser maior que a ordem de degasagem do submodelo. Fixando-se os valores de $r$ e $d$, o valor do AIC torna-se

$$
\text { (7) } \operatorname{AIC}\left(p_{1}, p_{2}, r, d\right)=-2 l(r, d)+2\left(p_{1}+p_{2}+2\right) \text {, }
$$

onde

$$
l(r, d)=-\frac{n-p}{2}\{1+\log (2 \pi)\}-\frac{n_{1}(r, d)}{2} \log \left\{\left(\hat{\sigma}_{1}(r, d)^{2}\right\}-\frac{n_{2}(r, d)}{2} \log \left\{\left(\hat{\sigma}_{2}(r, d)^{2}\right\}\right.\right.
$$

é o logaritmo da função de máxima verossimilhança que deve ser maximizada. $\mathrm{O}$ processo de maximização requer que algumas restrições sejam impostas, definindose, por exemplo, os percentis da base de dados, fixando-se os limites dentro dos quais o parâmetro de threshold será buscado.

Além disso, as variâncias dos resíduos $\sigma_{1}$ e $\sigma_{2}$ não necessariamente serão idênticas para os dois regimes, de modo que o SETAR poderá levar em conta a heterocedasticidade dos dados. Essa é uma propriedade importante do modelo, uma vez que é uma caracerística bem documentada na literatura da inflação brasileira.

Em particular, pelo critério do mínimo AIC, a melhor ordem é selecionada de forma tradicional, e cada submodelo é estimado por mínimos quadrados ordinários, empregando-se a base de dados pertencente a cada regime. Ademais, um estimador menos viesado para a variância dos erros pode ser obtido, normalizando-se a soma dos quadrados dos resíduos pelo tamanho efetivo da amostra. Assim, a variância não viesada dos resíduos $\left(\widetilde{\sigma}_{i}^{2}\right)$ do $i$-ésimo regime pode ser obtida a partir da seguinte expressão:

$$
\text { (9) } \tilde{\sigma}_{i}^{2}=\hat{\sigma}_{i}^{2} \frac{n_{i}}{n_{i}-p_{i}-1}
$$

onde $p_{i}$ é a ordem de defasagem do $i$-ésimo submodelo estimado. 


\section{Resultados e discussão}

Aplicando-se o critério do mínimino AIC, fixando os valores $\max (p=5 ; d=5)$ e definindo o invervalo de busca da threshold entre o $10^{\circ}$ e o $90^{\circ}$ percentil, foram obtidos os seguintes resultados. O objetivo é testar diferentes ordens e parâmetros de defasagem para o SETAR.

Tabela 4

AIC Nominal para o SETAR estimado para a taxa de inflação: $1 \leq d \leq 5$

\begin{tabular}{ccccc}
\hline$\hat{d}$ & AIC & $\hat{r}$ & $\hat{p}_{1}$ & $\hat{p}_{2}$ \\
\hline 1 & 3126 & 5,14 & 3 & 4 \\
2 & 3195 & 4,21 & 3 & 4 \\
3 & 3223 & 4,21 & 3 & 4 \\
4 & 3203 & 4,21 & 3 & 4 \\
5 & 3178 & 6,52 & 3 & 4 \\
\hline
\end{tabular}

Fonte: Elaboração própria.

Embora a ordem máxima de defasagem permitida tenha sido fixada em 5, pelo critério empregado, o melhor modelo é o de ordem 3 para o baixo regime e de ordem 4 para o alto regime. Esses resultados sugerem que o melhor SETAR que descreve a inflação brasileira é o $\operatorname{SETAR}(2 ; 3 ; 4)$ com $d=1$. A Tabela 5, abaixo, apresenta a estimativa dos parâmetros e também o diagnóstico dos resíduos pelo teste de Box e Pierce para a hipótese nula de ausência de autocorrelação na defasagem $(\cdot)$.

Tabela 5

$\operatorname{SETAR}(2 ; 3 ; 4)$ estimado para a taxa de inflação brasileira: 1944-2009 resultados

\begin{tabular}{c|c|c|c|c}
\hline & Estimativa & Erro padrão & Estatística $t$ & $p$-valor \\
\hline$\hat{d}$ & 1 & & \\
$\hat{r}$ & 5,14 & Baixo Regime $\left(n_{1}=603\right)$ & \\
\hline \multicolumn{5}{c}{} \\
\hline$\hat{\phi}_{1,0}$ & 0,382 & 0,073 & 5,258 & 0,000 \\
$\hat{\phi}_{1,1}$ & 0,733 & 0,044 & 16,701 & 0,000 \\
$\hat{\phi}_{1,2}$ & 0,022 & 0,044 & 0,497 & 0,619 \\
$\hat{\phi}_{1,3}$ & 0,051 & 0,022 & 2,253 & 0,025 \\
$\tilde{\sigma}_{1}^{2}$ & 1,334 & & & \\
\hline
\end{tabular}

Continua... 
Tavela 5 - Continuação

\begin{tabular}{cccccc}
\hline & Estimativa & Erro padrão & Estatística $t$ & $p$-valor \\
\hline$\hat{\phi}_{2,0}$ & 1,571 & 0,856 & 1,836 & 0,068 \\
$\hat{\phi}_{2,1}$ & 0,960 & 0,078 & 12,356 & 0,000 \\
$\hat{\phi}_{2,2}$ & $-0,010$ & 0,106 & $-0,091$ & 0,927 \\
$\hat{\phi}_{2,3}$ & $-0,244$ & 0,111 & $-2,1963$ & 0,029 \\
$\hat{\phi}_{2,4}$ & 0,177 & 0,080 & 2,222 & 0,027 \\
$\tilde{\sigma}_{2}^{2}$ & 48,176 & & & \\
BP(3) & 0,911 & & & \\
BP(6) & 0,933 & & \\
BP(12) & 0,249 & & \\
MAIC & 3128 & & \\
\hline Nota: BP (.) denota a ordem de defasagem do teste de Box e Pierce para autocorrelação \\
dos resíduos e o p-valor correspondente.
\end{tabular}

Em geral, para cada regime, a estatística $t$ e os correspondentes $p$-valores apresentados na Tabela 5, acima, indicam que os coeficientes estimados são estatisticamente signficativos. Observa-se que os coeficientes de defasagem de segunda ordem, nos dois regimes, não são significativos estatisticamente. Isso indica que o modelo pode ser reduzido a uma ordem inferior, em cada regime, embora esse procedimento não tenha sido adotado neste trabalho. A heterocedasticidade incondicional no regime de alta inflação é 35 vezes maior do que a verificada no regime de baixa inflação. Outro resultado interessante é que as probabilidades incondicionais, que medem a persistência de cada regime, são $\Pi_{1}=0,7674$ e $\Pi_{2}=0,2326$, indicando que o regime de baixa inflação tem uma probabilidade sensivelmente maior de ocorrência ${ }^{6}$.

(6) $\Pi_{i}=\frac{\mathrm{n}_{\mathrm{i}}}{\mathrm{n}}$, onde $\mathrm{n}$ é o tamanho total da amostra e $\mathrm{n}_{\mathrm{i}}$ é a quantidade de observações pertecente a cada regime. 
Figura 2

Separação dos regimes de baixa e alta inflação no Brasil - 1944-2009

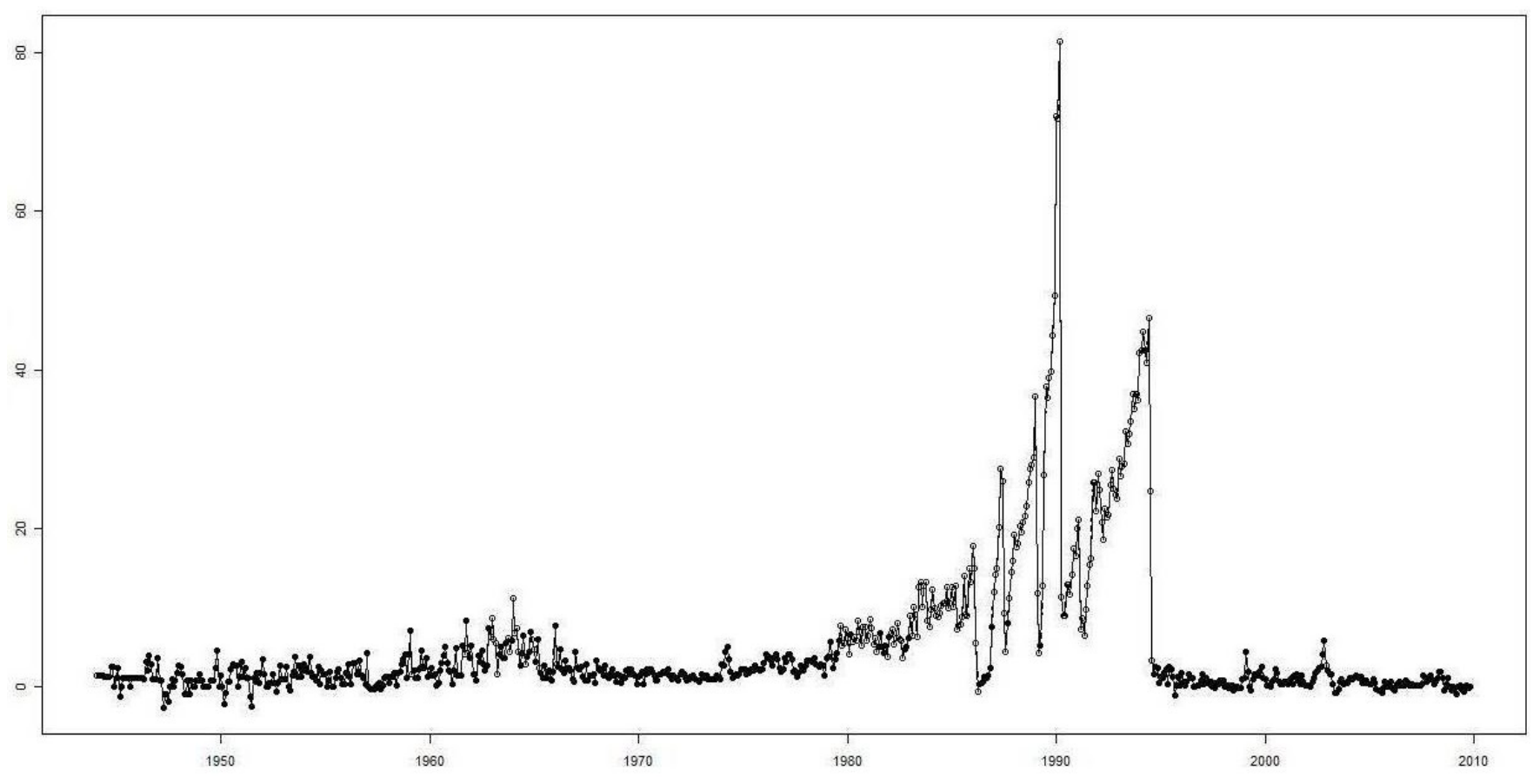


Como se esperava teoricamente, os coeficientes de primeira ordem de defasagem, em ambos os regimes, são estatisticamente significativos. No caso do regime de alta inflação, o valor do coeficiente está bem mais próximo da unidade, indicando o elevado grau de indexação da economia. Nesse sentido, o grau de indexação é função do nível de inflação. A threshold foi estimada em 5,14\%, situando-se aproximadamente no $77^{\circ}$ percentil da base de dados. Uma forma de explorar visualmente esses resultados é idenficando os regimes de alta e baixa inflação em sua trajetória temporal. De fato, isso foi feito separando-se as observações para a amostra completa, tal como aparece na Figura 2, acima.

Nessa figura, podemos identificar as realizações da série que pertence ao regime de baixa inflação, isto é, os valores que, com uma defasagem, são iguais ou menores que 5,14\%, identificados pelos círculos sólidos. Em contraste, as observações que pertencem ao regime de alta inflação no Brasil, cujos valores excedem o parâmetro de threshold, são representados pelos círculos vazios.

Observando-se a Figura 2, constata-se um padrão no comportamento da série. Em geral, o regime de baixa inflação corresponde à fase ascendente do processo inflacionário e vice-versa. Especialmente na década de 1990, parece claro que o baixo regime persiste na fase crescente do processo, e o alto regime predomina nos picos e na fase decrescente do processo, caracterizando uma evolução assimétrica na trajetória da inflação, uma vez que a fase de ascenção é mais lenta que a de decaimento. Esse fenômeno caracteriza a irreversibilidade no tempo. Dispondo as observações separadas em dois regimes diferentes, como na figura 2, acima, pode-se notar, com clareza, esse padrão de comportamento.

O SETAR $(2 ; 3 ; 4)$ estimado pode capturar a aceleração sucessiva da taxa de inflação e os planos de estabilização ao longo do tempo, desde a década de 1960 , sem tratar as observações pertencentes ao regime de hiperinflação como se fossem "atípicas" ou "outliers". Essa característica é típica da dinâmica não linear. Assim, pode-se concluir que uma alta taxa de inflação e, eventualmente, um período de hiperinflação podem ser partes integrantes de uma economia em desenvolvimento, e não um evento atípico ou exógeno como se costuma supor convencionalmente.

De fato, pode-se observar que, depois de esforços conservadores para estabilizar os preços com políticas de recessão e desemprego no início da década de 1980, alguns planos com base na hipótese de inflação inercial foram implementados, como o Plano Cruzado em 1986.

Posteriormente, ocorreram sucessivas tentativas de combate à inflação na economia brasileira. No entanto, após alguns planos mal sucedidos com base no congelamento (e descongelamento gradual) de preços e salários, com o Plano Real, em 1994, foi alcançada a estabilidade de preços. Basicamente, o Plano Real consistiu numa grande e complexa Reforma Monetária introduzida em estágios na 
economia, removendo-se a indexação de preços e salários pela introdução de uma nova unidade de medida indexada, simulando-se o estágio final de um processo de hiperinflação sem recessão ou desemprego ${ }^{7}$.

Como consequência, a característica interessante do SETAR estimado é a possibilidade de capturar o movimento assimétrico da série sem tratar a intervenção governamental e a mudança de regime (de alta para baixa inflação e volatilidade) como se fossem choques exógenos, pois a transição de um regime para outro pode ocorrer sempre que o limiar de 5,14\% ao mês for ultrapassado. Ademais, em um processo de hiperinflação, em que as políticas fiscal e monetária são passivas (Dornbusch et al., 1990), o processo pode ser alterado endogenamente pelos agentes econômicos sem mudança abrupta nas políticas fiscais e monetárias. Essa dinâmica endógena da inflação foi pioneiramente descrita por Kaldor (1982, p. 61) e, mais tarde, formalizada por Lopes (1985). A abordagem linear das séries temporais, em geral, trataria as observações da hiperinflação como se fossem “aberrantes" ou "outliers". Não é esse o caso na abordagem não linear".

Parece ter sido essa a interpretação de Cati et al. (1999) no contexto dos testes de raiz unitária em que tratam a taxa de inflação brasileira como se fosse uma série linear e reversível no tempo. Os autores assumem, em seu teste de raiz unitária, que a taxa de inflação brasileira pode ser gerada (apenas) por uma combinação linear de seus valores presentes e passados sem considerar a possibilidade de uma dinâmica não linear'. As evidências aqui apresentadas sugerem que essa interpretação é de difícel sustentação porque o processo não linear pode ter uma dinâmica endógena que transcende a intervenção governamental $^{10}$. Esse pode ter sido também o caso da hiperinflação na Alemanha no início dos anos 1920. Essa observação é consistente com os resultados apresentados neste trabalho e também com os modelos teóricos analisados em Lopes (1985), Dornbusch et al. (1990) e Serrano (2006).

Além do teste para os resíduos, o modelo ajustado pode ser avaliado analiticamente ou por simulação. O método escolhido depende, entre outras questões, da ordem de defasagem do modelo estimado. Se o modelo estimado possui defasagem de primeira ordem, é possível obter a condição de estabilidade

(7) Desde sua origem, os inercialistas apresentaram duas propostas alternativas de combate à inflação. Inicialmente, foi sugerida a política de congelamento (com descongelamento gradual) de preços e salários acompanhada pelo congelamento de alguns preços-chave da economia. A alternativa do congelamento era realizar uma complexa Reforma Monetária, simulando o estágio final de um processo de hiperinflação. Ver Lopes (1985), Resende (1985a, 1985b) e Bacha (1997).

(8) Ver Franses e Dijk (2003) para uma discussão detalhada.

(9) Ver, por exemplo, as equações (15) e (16) de Cati et al. (1999), que representam o teste ADF modificado pelos autores. Essa modificação foi realizada com a introdução de dummies exógenas no teste Aumentado de Dickey-Fuller.

(10) No caso do Plano Real, realizada sem choque ou congelamento de preços e salários. 
analiticamente, mas não é esse o caso da inflação brasileira. Para os modelos de ordem superior à unidade, é extremamente difícil estabelecer analiticamente a condição para equilíbrios estáveis, atratores e/ou limit cycles. Uma forma de proceder é seguir Franses e Dijk (2003), fazendo uma simulação determinística a partir do skeleton do modelo, abandonando o termo de erro. No entanto, a opção adotada foi fazer uma simulação estocástica, seguindo Cryer e Chan (2008), uma vez que o número de observações e seu comportamento podem ser facilmente dispostos graficamente. Com o termo de erro no modelo, o comportamento da taxa de inflação simulada para a economia brasileira a partir do modelo $\operatorname{SETAR}(2 ; 3 ; 4)$ estimado é apresentado na Figura 3.

Figura 3

Simulação estocástica da taxa de inflação brasileira a partir do $\operatorname{SETAR}(2 ; 3 ; 4)$ estimado

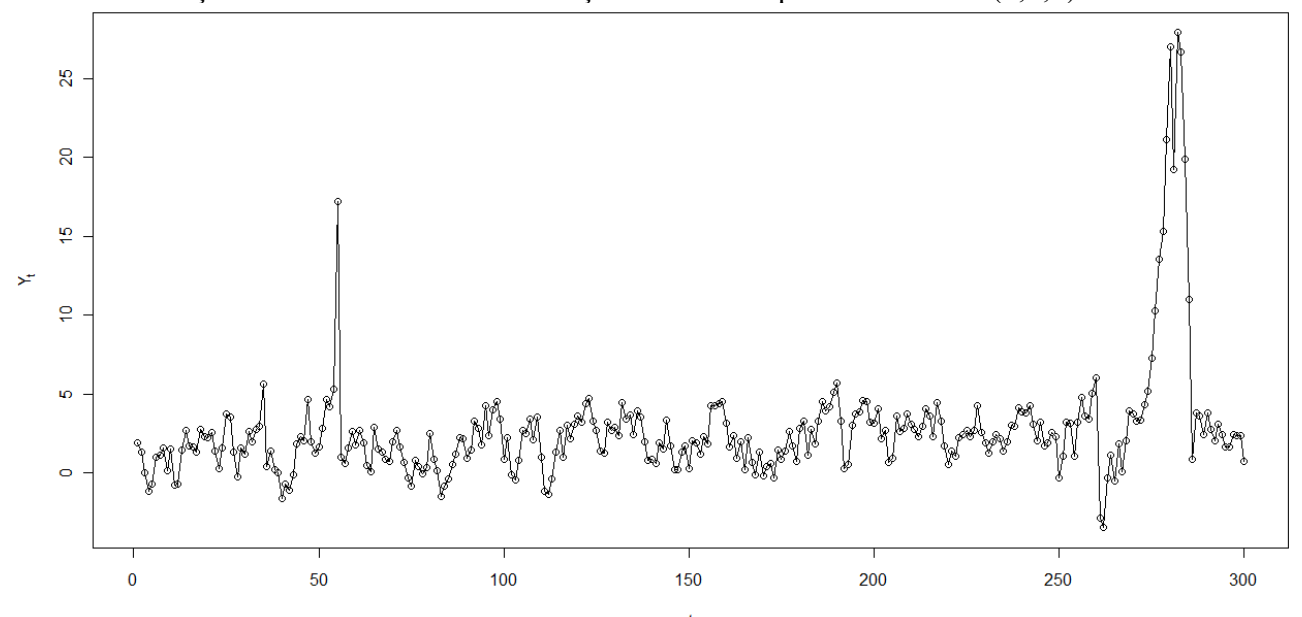

A Figura 3, acima, mostra uma realização típica do modelo SETAR a partir dos coeficientes estimados, apresentados na Tabela 5. Observa-se que os valores simulados reproduzem o comportamento assimétrico da inflação, em que a ascensão é lenta e seu decaimento é mais rápido; todavia, seu comportamento em torno de uma média sugere que a série simulada é estacionária.

Apesar de vigorar a interpretação corrente de que, na economia brasileira, predomina a alta inflação quando se consideram longos períodos de tempo, desde a fase inicial de sua industrialização, os resultados indicam que o regime de alta inflação predominou apenas em alguns subperíodos específicos, em particular na década de 1960 e nas últimas duas décadas perdidas, ou seja, 1980 e 1990. Adicionalmente, observa-se que a cada regime corresponde um grau particular de volatilidade da inflação.

A Figura 3 registra também a possibilidade de ocorrência de uma eventual inflação elevada sem que o processo se torne permanente, mas que tenha caráter 
transitório na economia, pois o sistema econômico poderá oscilar entre a alta e a baixa inflação. A despeito desse comportamento, a taxa média de inflação poderá se estabilizar em algum patamar positivo no regime de baixa inflação sem ultrapassar o limiar, com baixa volatilidade. Por isso, pode-se concluir que a ocorrência de uma alta taxa de inflação na economia brasileira não constitui um acontecimento atípico, como alguns autores tendem a interpretar, mas um fenômeno (endógeno) state-depedent na economia, uma vez que o grau de indexação depende do nível de inflação. Quando a taxa de inflação se aproxima do limiar, os agentes econômicos demandam mais indexação (formal e informal); desse modo, a mudança para o regime de alta inflação e volatilidade é induzida pelos próprios agentes econômicos ${ }^{11}$.

Esses resultados reforçam a necessidade de a política econômica manter o regime atual de baixa inflação e baixa volatilidade na economia brasileira, seja pela expansão da produção de mercadorias, seja reduzindo os custos (incluindo taxas de juros) e pela remoção gradual de alguns resíduos de inércia, uma vez que alguns estudos, como o de Figueiredo e Marques (2009), sugerem que o excesso de demanda não é uma causa primária da inflação no Brasil. De acordo com Dornbusch et al. (1990) e em consonância com os resultados deste trabalho, a importância e a eficácia das políticas monetária e fiscal ativas para controlar a inflação dependem do regime que vigora na economia. No regime de baixa inflação, a experiência tem mostrado que essas políticas são eficazes e que podem manter o regime por um longo período de tempo.

\section{Conclusões}

O objetivo principal do estudo foi investigar assimetrias e não linearidades no comportamento da inflação brasileira, descrevendo-a como um processo autorregressivo sujeito a mudanças de regime. A metodologia consistiu na aplicação de dois testes para a não linearidade quadrática e um teste com alternativa específica para o tipo threshold a partir de uma base de dados mensal para o período de fevereiro de 1944 a novembro de 2009. A seguir, foi estimado um modelo SETAR com dois regimes capaz de descrever o comportamento não linear e assimétrico de um processo autorregressivo.

Todos os testes indicaram a não linearidade da inflação brasileira. Os resultados das estimações sugerem a ocorrência de pelo menos dois regimes distintos com diferentes processos geradores. O regime de baixa inflação é o menos volátil e o mais persistente, com alta probabilidade de ocorrência. A despeito dos períodos de alta inflação e alta volatilidade, conclui-se que o regime de baixa inflação com baixa volatilidade é uma característica predominante na economia

(11) Esse comportamento dos agentes econômicos é inteiramente compatível com o postulado da racionalidade. Ver Lopes (1985) para uma discussão a partir do dilema dos prisioneiros. 
brasileira. A simulação a partir dos coeficientes do SETAR estimado sugeriu a estacionariedade da série. A análise dos resultados sugere que o $\operatorname{SETAR}(2 ; 3 ; 4)$ descreve razoavelmente o comportamento de longo prazo da taxa de inflação brasileira.

Com a estimação do SETAR, foi possível estabelecer o limiar para a taxa de inflação brasileira: 5,14\%, cujo valor separa os regimes de baixa e alta inflação e volatilidade no Brasil. O regime de baixa inflação é caracterizado por uma baixa taxa de inflação mensal, com volatilidade 35 vezes menor do que o regime de alta, e está associado à alta probabilidade de ocorrência (alta persistência). Outra característica notável desse regime é o menor grau de indexação da economia, uma vez que o coeficiente autorregressivo de primeira ordem é sensivelmente mais baixo quando comparado com o regime de alta inflação. Em contraste, o regime de alta inflação é caracterizado por uma taxa de inflação acima de $5,14 \%$ ao mês e pela presença de uma componente inercial substancialmente maior expressa pelo coeficiente autorregressivo de primeira ordem desse regime. Além disso, foi possível constatar que este último regime tem baixa probabilidade de ocorrência, sugerindo a baixa persistência do regime de alta inflação no Brasil.

Uma vez que a persistência do regime de baixa inflação é maior, espera-se que o regime de inflação baixa perdure por longos períodos de tempo. Todavia, como há evidências de dinâmica não linear (state-dependent) para controlar e manter o regime vigente de baixa inflação e volatilidade na economia, há explícita necessidade de políticas fiscais e monetárias ativas. Caso contrário, o processo inflacionário poderia assumir uma dinâmica própria induzida pelo comportamento defensivo dos agentes econômicos e, assim, mudar para o regime de alta inflação e volatilidade endogenamente, uma vez que a threshold seja ultrapassada.

Há evidências de instabilidade intrínseca na economia brasileira, exigindo, assim, a mão visível do Estado para sua estabilização e controle. Em particular, as evidências reforçam a importância dos "fatores de estabilização" listados por Dornbusch et al. (1990), como as políticas monetária e fiscal ativas e a remoção da indexação de salários e preços para evitar espirais inflacionárias. Nessa direção, pode-se concluir que o limiar para a inflação brasileira postulado por Dornbusch et al. (1990) é sensivelmente maior do que a estimativa encontrada neste estudo; ao contrário do senso comum, os resultados sugerem que a tolerância ao processo inflacionário no Brasil é baixa, não alta.

Havendo inércia significativa no regime de baixa inflação, sugere-se expurgar os resíduos (formais e informais) de indexação que persistem na economia brasileira, reduzindo-se, assim, a componente inercial, isto é, o grau de indexação. Essa seria uma estratégia de política mais salutar do que provocar recessão e desemprego através da restrição ao crédito. Uma lição é clara: a estabilidade dos preços na economia brasileira está longe de ser automática. 


\section{Referências bibliográficas}

BACHA, E. L. O Plano Real: uma avaliação. In: MERCADANTE, A. (Org.). O Brasil pós-Real: a política econômica em debate. Campinas: Unicamp-IE, 1997. p. 11-69.

CAMPÊLO, A. K.; CRIBARI-NETO, F. Inflation inertia and 'inliers': the case of Brazil. Revista Brasileira de Economia, v. 57, n. 4, p. 713-719, 2003.

CATI, R. C.; GARCIA, M. G. P.; PERRON, P. Unit roots in the presence of abrupt governmental interventions with an application to Brazilian data. Journal of Applied Econometrics, 14, p. 27-56, 1999.

CHAN, K. S. Testing for threshold autoregression. The Annals of Statistics, v. 18, n. 4, p. 1886-1894, 1990.

Percentage points of likelihood ratio tests for threshold autoregression. Journal of Royal Statistical Society, Series B, v. 53, n. 3, p. 691-696, 1991.

CRIBARI-NETO, F.; CASSIANO, K. Uma análise da dinâmica inflacionária brasileira. Revista Brasileira de Economia, v. 59, n. 4, p. 535-566, 2005.

CRYER, J. D.; CHAN, K. S. Time series analysis. New York: Springer, 2008.

DELFIM NETTO, A. O Plano Real e a armadilha do crescimento econômico. In: MERCADANTE, A. (Org.). O Brasil Pós-Real: a política econômica em debate. Campinas, SP: Unicamp/IE, 1997. p. 89-100.

DORNBUSCH, R.; STURZENEGGER, F.; WOLF, H. Extreme inflation: dynamics and stabilization. Brooking Papers on Economic Activity, v. 2, p. 1-84, 1990.

EATWELL, J.; MILGATE, M. (Ed.). Keynes's economics and the theory of value and distribution. London: Duckworth, 1983.

ELLIOT, E.; KIEL; L. D. Introduction. In: ELLIOT, E.; KIEL; L. D. (Ed.). Chaos theory in the social sciences. Foundations and applications. Michigan: Michigan University Press, 2004. p. 1-15.

FIGUEIREDO, E. A.; MARQUES, A. M. Inflação inercial como um processo de longa memória: análise a partir de um modelo arfima-figarch. Estudos Econômicos, v. 39, n. 2, p. 437-458, 2009.

FRANSES, P. H.; van DIJK, D. Nonlinear time series models in empirical finance. Cambridge: Cambridge University Press, 2003.

HAMILTON, J. A new approach to the economic analysis of nonstationary time series and business cycle. Econometrica, v. 57, n. 2, p. 357-384, 1989.

KALDOR, N. The scourge of monetarism. London: Oxford University Press, 1982.

KEENAN, D. A Tukey nonlinear type test for time series nonlinearities. Biometrika, 72, p. 39-44, 1985.

KEYNES, J. M. The general theory of employment, interest and money. London: Macmillan, 1936. 
LAURINI, M.; VIEIRA, H. A dynamic econometric model for inflationary inertia in Brazil. 2005. (Working Paper). Disponível em: http://www.mlcc.com.br/artigos/inertiaem.pdf. Acesdo em: 7 mar 2008.

LÉON-LEDESMA, M. A. Cycles, aggregate demand, and growth. In: ARESTIS, P.; McCOMBIE, J.; VICKERMAN, R. (Ed). Growth and economic development. Cheltenham, UK: Edward Elgar Publishing, 2006. p. 82-95.

LIU, T.; GRANGER, C. W. J.; HELLER, W. P. Using the correlation exponent to decide whether an economic series is chaotic. Journal of Applied Econometrics, 7 (Supplement), S25-S39, 1992.

LOPES, F. L. Inflação inercial, hiperinflação e desinflação: notas e conjecturas. Revista de Economia Política, v. 5, n. 2, p. 135-151, 1985.

; WILLIAMSON, J. A teoria da indexação consistente. Estudos Econômicos, v. 10, n. 3, p. 61-99, 1980.

MAIA, A. L. S.; CRIBARI-NETO, F. Dinâmica inflacionária brasileira: resultados de auto regressão quantílica. Revista Brasileira de Economia, v. 60, n. 2, p. 153-165, 2006.

MODIANO, E. A dinâmica de salários e preços na economia brasileira: 1966-1981. Pesquisa e Planejamento Econômico, v. 13, n. 1, p. 39-68, 1983.

- Salários, preços e câmbio: os multiplicadores dos choques numa economia indexada. Pesquisa e Planejamento Econômico, v. 15, n. 1, p. 1-32, 1985.

PESARAN, M. H.; POTTER, S. M. Nonlinear dynamics and econometrics: an introduction. Journal of Applied Econometrics, v.7 (Supplement: Special Issue), p. S1-S7, 1992.

POTTER, S. M. A nonlinear approach to US GNP. Journal of Applied Econometrics, v. 10, n. 2, p. 109-125, 1995.

PROIETTI, T. Characterizing asymmetries in business cycles using smooth-transition structural time series models. Studies in Nonlinear Dynamics and Econometrics, 3, p. 141$156,1998$.

REISEN, V. A.; CRIBARI-NETO, F.; JENSEN, M. J. Long memory inflationary dynamics: the case of Brazil. Studies in Nonlinear Dynamics \& Econometrics, v. 7, n. 3, p. $1-16,2003$.

RESENDE, A. L. A moeda indexada: nem mágica nem panacéia. Revista de Economia Política, v. 5, n. 2, p. 124-129, 1985a.

A moeda indexada: uma proposta para eliminar a inflação inercial. Revista de Economia Política, v. 5, n. 2, p. 130-134, 1985 b.

SERRANO, F. Mind the gap: hysteresis, inflation dynamics and the Sraffian supermultiplier. 2006. Disponível em: http://www.ie.ufrj.br/datacenterie/pdfs/ download/texto_10_10.pdf. Acesso em: 10 jun 2010.

TEJADA, C. A. O.; PORTUGAL, M. S. Credibilidade e inércia inflacionária no Brasil: 1986-1998. Estudos Econômicos, v. 31, n. 3, p. p. 459-494, 2001. 
THE R DEVELOPMENT CORE TEAM. $R$ : A language and environment for statistical computing. Vienna, Austria: R Foundation for Statistical Computing, 2008. Disponível em: http://www.r-project.org.

TIAO, G. C.; TSAY, R. S. Some advances in non-linear and adaptive modeling in time series. Journal of Forecasting, 13, p. 109-131, 1994.

TONG, H. Threshold models in non-linear time series analysis. New York: Springer, 1983. p. 101-141. . Non-linear time series. Oxford: Clarendon Press, 1990.

; LIM, K. S. Threshold autoregression, limit cycles and cyclical data (with discussion). Journal of the Royal Statistical Society B, 42, p. 245-292, 1980.

TSAY, R. S. Nonlinearity tests for time series. Biometrika, 73, p. 461-466, 1986.

. Detecting and modeling nonlinearity in univariate time series analysis. Statistica Sinica, 1, p. 431-451, 1991. 